\title{
ANÁLISIS DE FACTORES DE EMPODERAMIENTO DE MAESTROS ESPECIALISTAS EN LA ENSEÑANZA INCLUSIVA DE NIÑOS DE 6-16 AÑOS CON DIFICULTADES AUDITIVAS
}

\author{
Olga María Alegre de la Rosa \\ Universidad de La Laguna \\ Luis Miguel Villar Angulo \\ Universidad de Sevilla
}

\begin{abstract}
RESUMEN: Este estudio ha analizado los factores de empoderamiento de maestros tutores sobre la enseñanza inclusiva de niños de 6-16 años con dificultades auditivas. La muestra del estudio estaba compuesta por 297 maestros tutores de Gran Canaria y Tenerife. Se utilizó el Cuestionario Incluye (Cl) basado en el Index for Inclusion, compuesto por tres dimensiones: Cultura, Política y Prácticas Inclusivas. El análisis primario incluyó un análisis factorial exploratorio. Los resultados indicaron que un empoderamiento docente para la integración de niños con dificultades auditivas en centros inclusivos está sustentado por siete factores: Desarrollo profesional (DP), Autoeficacia en la enseñanza de destrezas, Liderazgo inclusivo, Gestión de la tecnología, Retos de implicación familiar, Marcos de pensamiento experto, y Usabilidad tecnológica y accesibilidad universal. Estos hallazgos implican delinear cambios curriculares en el grado de Maestro. La inclusión se convierte en el valor nuclear de empoderamiento en las competencias básicas y transversales de las materias del grado académico de Maestro. Asimismo, recomendamos el desarrollo de estándares profesionales y cursos de formación docente para la educación inclusiva de niños con dificultades auditivas.
\end{abstract}

PALABRAS CLAVE: Factores de empoderamiento docente, prácticas inclusivas, niños con dificultades auditivas, análisis factorial.

\section{ANALYSIS OF THE EMPOWERMENT FACTORS OF SPECIALIST TEACHERS IN THE INCLUSIVE EDUCATION OF CHILDREN AGED 6-16 WITH HEARING DIFFICULTIES}

\footnotetext{
ABSTRACT: This study has analysed the factors of empowerment of tutor teachers on the inclusive education of 6-16-year-old children with hearing
} 
difficulties. The study sample consisted of 297 teachers from Gran Canaria and Tenerife. The Inclusion Questionnaire (IQ) was used based on the Index for Inclusion, composed of three dimensions: Culture, Politics and Inclusive Practices. The primary analysis included an exploratory factor analysis. The results indicated that teacher empowerment for the integration of children with hearing difficulties in inclusive schools is supported by seven factors: professional development (DP), self-efficacy in the teaching of skills, inclusive leadership, technology management, family involvement challenges, expert thinking frameworks, and technological usability and universal accessibility. These findings imply delineating curricular changes for the Teacher degree. Inclusion becomes the core value of empowerment in the primary and transversal competencies of the subjects of the teacher certification degrees. Besides, we recommend the development of professional standards and teacher training courses for the inclusive education of children with hearing difficulties.

KEYWORDS: Factors of teacher empowerment, inclusive practices, children with hearing difficulties, factor analysis.

Recibido: $26 / 11 / 2018$

Aceptado: 08/06/2019

Correspondencia: Olga María Alegre de la Rosa, Universidad de La Laguna, C/ Heraclio Sánchez, 43, 38204 San Cristóbal de La Laguna, Santa Cruz de Tenerife. Email: oalegre@ull.es.

\section{INTRODUCCIÓN}

La educación inclusiva (EI) es un proceso de fortalecimiento de la capacidad del sistema educativo para conseguir la estrategia Educación para Todos, según la Declaración de Salamanca (UNESCO, 1994). Tras la declaración, muchos investigadores han dilatado conceptualmente la El afectando su campo de intervención. Recientemente, los supuestos formales en que se basa la El han sido metaanalizados por Van Mieghem, Verschueren, Petry y Struyf (2018, p. 10), configurando su esencia, tras revisar 26 estudios, en los siguientes principios: "(1) actitudes hacia la El; (2) desarrollo profesional de los docentes que fomenta la El; (3) prácticas que mejoran la El y (4) participación de estudiantes con necesidades educativas especiales (NEE)". A partir de este metaanálisis, ensayamos la siguiente revisión de la literatura para este artículo.

\section{Actitudes y autoeficacia hacia la EI}

Una revisión de 26 estudios hecha por De Boer, Pijl y Minnaert (2011) había revelado que la mayoría de los maestros tenían actitudes neutrales o negativas hacia la inclusión de alumnos con necesidades especiales en educación primaria. Ninguno de los estudios informó de resultados positivos claros. Tampoco pudieron extraer 
conclusiones sobre los efectos de las actitudes docentes hacia la participación social de los alumnos con necesidades especiales. Tras aislar variables problemáticas, los autores hallaron actitudes promisorias de los docentes, como el adiestramiento, la experiencia en la El y el tipo de discapacidad de los alumnos.

En este estado de divagación, Özokcu (2018, p. 7) aportó estudios concluyentes sobre esta cuestión con la aseveración fundada de que "los docentes ni tenían una actitud negativa ni positiva hacia la educación inclusiva". En este sentido, la autoeficacia de maestros en colaboración con colegas ha augurado más creencias y sentimientos positivos hacia la inclusión de estudiantes con dificultades en su desarrollo y, simultáneamente, ha predicho menos preocupaciones de los maestros sobre la inclusión de esta población de estudiantes (Montgomery y Mirenda, 2014).

\section{Desarrollo profesional docente que fomenta la EI}

La situación de la El en la formación inicial de muchos países ha tenido que dar un giro para incorporar programas específicos que aborden con rigor la "detección, discriminación, identificación, y comprensión" de niños con dificultades sensoriales, en particular, niños con implantes auditivos en educación musical (Schraer-Joiner y Prause-Weber, 2009).

Cuando el personal de los centros educativos no estaba capacitado para responder a las necesidades de los niños implantados con prótesis auditivas (cocleares o audífonos) y a las demandas educativas de los padres, las instituciones administrativas habilitaron cursos de perfeccionamiento que cubrían las dificultades auditivas, como patentizaron en su estudio Wilson, Ellen y Houston (2010).

La cuestión más valiosa del conocimiento de los maestros de los centros educativos es la autorreflexión sobre el alcance de sus destrezas. La investigación de las creencias de los maestros en torno a la El dista de un conocimiento empíricamente demostrado de la misma. Sin embargo, una autoevaluación de sus disposiciones influye las actitudes y creencias hacia la práctica de la El (Zagona, Kurth y MacFarland, 2017). Porque los dos factores que inmovilizan el desarrollo profesional docente, en palabras de Sprott (2019, p. 325), son "Ios obstáculos estructurales que impiden las oportunidades de colaboración y los rígidos mandatos jerárquicos". En particular, este autor subraya este último impedimento de subordinación que va en contra de una "enseñanza inclusiva, individualizada y centrada en el estudiante".

\section{Prácticas y recursos humanos que mejoran la EI}

Un proyecto educativo de centro asegura como principio ineludible el apoyo a las familias para la detección auditiva y la intervención coordinada con pediatras especializados en niños con dificultades sensoriales. Hay un principio de justicia social consensuado entre países: los programas educativos de los centros "utilizarán diversas estrategias para ayudar a las familias a comprender la importancia de un oportuno seguimiento temporal" (Moeller, Carr, Seaver, Stredler-Brow y Holzinger, 2013, p. 430). 
Una de las estrategias de ayuda es la utilización de un "liderazgo integral que reside en la intersección de varios espacios de actividad para el desarrollo institucional" (Black y Simon, 2014, p. 156). Es decir, un liderazgo entendido como incitación a la satisfacción de necesidades especiales de niños en unidades organizativas con apoyos amplios y flexibles donde los estudiantes tengan libertad de creación y recreación de sí mismos.

La evolución de las ideas y la semántica del liderazgo nos han conducido a un nuevo término que refleja la sensibilidad moral del empleado y la seguridad del paciente. Es el liderazgo inclusivo, Ileno de evocaciones de liderazgo transformacional, que encierra en su inspiración a la persona en el contexto de la diversidad, la cultura de la organización y la innovación (León, Crisol y Moreno, 2018). Porque en el liderazgo inclusivo lo que importa es el mundo del empoderamiento y el desarrollo de las personas, en fin, la posibilidad de ganar profundidad en la autonomía (Amin, Till y McKimm, 2018).

La confianza es el recurso esperado en las relaciones organizativas de un centro para que un maestro comparta responsabilidad con la dirección y equipo docente en el tratamiento de las dificultades académicas (prioritariamente del lenguaje) de los niños con dificultades auditivas "consultando a profesionales de otras áreas de discapacidad (ceguera, discapacidad intelectual y autismo)" (Bruce, Dinatale y Ford, 2008, p. 375).

La confianza es una de las potencias más poderosas de empoderamiento. Cuando penetra en el deseo profesional de un maestro, extrae de su introspección un poderío para rescatar del fondo de las interacciones acontecidas en el aula y centro, mundos sin restricciones, con acceso a todas las oportunidades, participación y éxito de niños con dificultades sensoriales en programas educativos culturalmente receptivos.

Desde el punto de vista de la política educativa, la legislación orientada a la educación de niños con dificultades sensoriales ha primado objetivos que representan el orden de las personas frente al desorden de la naturaleza: igualdad de oportunidades, participación plena (empoderamiento), vida independiente, autosuficiencia económica, sin que estas metas menoscaben la equidad como categoría de la humanidad donde el género, las creencias, la lengua, o la cultura se alinean para "predecir patrones esperados de estudiantes (rendimiento, participación, o capacidad para analizar críticamente datos o la sociedad)" (Moran y Larwin, 2017, p. 156).

En el mundo empresarial, compuesto de deseo y sueño, el autoempleo es la microempresa de un trabajador -universo laboral de nuestros días- que adquiere grandeza cuando se postula como objetivo para un proyecto de El. Cuando reparamos en este principio, un maestro está sentando el fundamento de un aprendizaje empresarial en un proyecto curricular, es decir, está identificando "oportunidades para crear o liberar valor" de las cosas (Rae y Carswell, 2001, p. 152); está dando inusitada importancia a actos y tareas que luego un estudiante con dificultades sensoriales, ya adulto, puede realizar desde casa para explotar oportunidades valiosas de generación de riqueza. 


\section{Participación de estudiantes con dificultades auditivas en la EI}

Los sistemas educativos de muchos países acogen normas y modelos de educación inclusiva. La inclusión no solo afecta el ámbito escolar sino a los espacios y personas que se relacionan con el aprendizaje fuera del centro escolar, subrayando la dimensión social de la educación. Así, pues, la El incluye diálogos multicanales entre profesores y sus cuadros directivos, entre niños y el personal de apoyo de atención primaria, y entre profesores, niños y padres (Jachova y Karovska, 2008). También incorpora, dependiendo de las necesidades del niño, diálogos del estudiante con un fisioterapeuta, un terapeuta ocupacional, o distinto personal de las subespecialidades médicas (psiquiatra, neurólogo, pediatra y / o educador con experiencia en sordera / ceguera). Estos diálogos abogan por el establecimiento de estrategias curriculares y didácticas variadas, y el desarrollo profesional de maestros para que conozcan procesos de intervención en las aulas de centros escolares (Jachova y Kovacevic, 2010).

Se ha comprobado que el aprendizaje de una palabra se abre en la mente de un niño conforme la palabra se usa en diversos contextos. El estudio de tres procesos de aprendizaje de la palabra -"fast mapping", retención, y extensión- por niños implantados permitió a Walker y McGregor (2013) concluir que esos niños demostraron déficits de aprendizaje de palabras, siendo especialmente problemático para ellos la retención de las mismas, cuando se compararon con compañeros sin implantes. Cuando los niños recibían un implante coclear (IC) en el segundo año de su vida, ¿qué efecto tenía en la comprensión del lenguaje y la producción? Esta pregunta fue la génesis del estudio de Caselli, Rinaldi, Varuzza, Giuliani y Burdo (2012), quienes, tras varias comparaciones de muestras de 17 niños italianos (oyentes, sordos, sordos con IC unilateral y bilateral, etc.), comprobaron el progreso en las destrezas del lenguaje de los niños sordos cuando se activó el IC en el segundo año de sus vidas, aunque persistieran ciertas debilidades fonológicas y morfosintácticas.

Otros investigadores han querido conocer el impacto del IC en la educación práctica de los niños sordos o con dificultades auditivas en los procesos de integración educativa, comunicación, opciones educativas y rendimiento académico, y han comprobado que, efectivamente, el IC temprano en la vida de los niños sordos o con dificultades auditivas había mejorado el lenguaje hablado y el rendimiento (Archbold y Mayer, 2012). No obstante, estos mismos autores habían apuntado los retos que tenía una El en aspectos de desarrollo profesional docente, manejo de recursos y nuevas tecnologías, y creación y mantenimiento de un ambiente físico adecuado. En efecto, Davenport y Alber-Morgan (2016) habían Ilamado la atención sobre ciertos aspectos constructivos de los edificios que incrementaban la rumorosidad en la comunicación y disminuían la atención de los niños con dificultades de audición: "pisos de baldosas, muros de hormigón, y grandes ventanas que reverberan el sonido. Además, un ambiente ruidoso, como el zumbido de los ordenadores, y los sistemas de aire acondicionado contribuyen a una difícil audición ambiental" (p. 44).

El rendimiento de los niños sordos con IC ha sido profusa y profundamente revisado por Mayer y Trezek (2018). Su informe, tras una revisión de 21 estudios en un periodo de 20 años, es concluyente para el conocimiento y mejora de la lectura y escritura de ese colectivo de niños. La comprensión lectora fue un efecto medido 
y conseguido por la mayor parte de los más de 1000 niños participantes en los diversos estudios, aunque dada la cantidad de investigaciones revisadas, existía variabilidad entre los hallazgos obtenidos. El estudio australiano de Punch y Hyde (2011) había tenido como sujetos de investigación a padres, maestros y niños, y relató experiencias positivas del desarrollo infantil, al tiempo que expuso las dificultades de comunicación de los sujetos en grupo y los apuros asociados con destrezas sociales.

El seguimiento de los niños con dificultades auditivas en las aulas ordinarias es un objetivo curricular de centros con aulas inclusivas de niños pequeños, mientras que la implicación de los adolescentes con doble IC es incuestionable para padres y maestros que subrayan la necesidad de que los estudiantes demuestren conductas de perseverancia para que se produzca una rehabilitación oportuna, aunque el esfuerzo psicológico sea más familiar que docente. Así lo constataron Mather, Archbold y Gregory (2011) que adujeron en su estudio el escaso número de centros educativos que ayudaban a los adolescentes en el proceso de transición a un doble IC, aunque valoraron los trabajos de logopedas en las sesiones individuales de audición. Cuando Vermeulen, De Raeve, Langereis y Snik (2012) aplicaron el "Screening Instrument for Targeting Educational Risk (SIFTER)" a niños holandeses comprobaron que sus necesidades se resolvían rápidamente, mientras que el desarrollo de las habilidades de comunicación eran más lentas, quizás debido al emplazamiento de los alumnos en espacios educativos ruidosos que deberían tener presentes los maestros de estudiantes implantados cuando ofrezcan apoyo de aprendizajes académicos.

\section{Materiales y MÉtodos}

\section{Participantes}

Los 297 participantes enseñaban en centros públicos de las islas Gran Canaria y Tenerife. La mayoría de los docentes eran mujeres $(n=265,82.2 \%)$. En mucha menor proporción, hombres $(n=32,10.8 \%)$. En el conjunto de la muestra, la edad dominante se situaba en el ciclo de 50-59 años $(n=202,68 \%)$, seguido del ciclo de $30-39$ años $(n=41,13.8 \%)$ y 40-49 años $(n=36,12.1 \%)$. A la luz del ciclo de edad mayoritario de los maestros, se puede considerar que estaban en la fase profesional de autoridad otorgada a sí mismo y de auto transformación (Maskit, 2011). Tenía la muestra una dilatada experiencia profesional: 250 participantes (84.2\%) acreditaron $10 \mathrm{o}$ más años de docencia activa, y a continuación 24 docentes (8.1\%) acreditaron entre 7 y 9 años de experiencia docente. En cuanto a su experiencia con niños de dificultades auditivas, la mayoría tenía 3 o menos años de experiencia profesional ( $n=187,63 \%)$, seguido del grupo docente que tenía entre 7 y 9 años $(n=65,21.9 \%)$.

En total, 297 niños y adolescentes con dificultades auditivas participaron en el estudio. Sus edades variaron entre 13-16 años de edad cronológica ( $n=100), 6-9$ años $(n=102)$ y $10-12$ años $(n=95)$. Había más niños $(n=160)$ que niñas $(n=137)$. Desde el punto de vista clínico, predominaron los niños y niñas que usaban IC $(n=187)$ frente a los que utilizaban audífonos (AUD) $(n=110)$. Cursaban Educación Primaria $(\mathrm{n}=182)$, Educación Secundaria Obligatoria $(\mathrm{n}=104)$, y Bachillerato o Formación Profesional $(n=11)$. Muchos niños y adolescentes no participaron de Adaptaciones 
Curriculares Individualizadas (ACIs) ( $\mathrm{n}=178$ ), frente a los que sí tenían ACls ( $\mathrm{n}=$ 119). El sistema de comunicación de los estudiantes con dificultades auditivas con sus familias era oral $(n=212)$, bilingüe $(n=57)$ o por lengua de signos $(n=28)$.

\section{Procedimiento}

Los investigadores contactaron por primera vez con la Consejería de Educación del Gobierno Insular para solicitar la cooperación de las escuelas de El que tuvieran niños con dificultades auditivas. Los maestros tutores de aula fueron seleccionados mediante muestreo de conveniencia, bajo la premisa de que tenían en sus aulas algún estudiante con dificultades auditivas y que estaban dispuestos a cooperar con los investigadores.

\section{Instrumentación}

Se construyó el Cuestionario Incluye $(\mathrm{Cl})$ a través de tres pasos. Primero, los investigadores buscaron palabras clave en el Index for Inclusion (Booth y Ainscow, 2000) y otras fuentes (por ejemplo, investigaciones recopiladas en ERIC) que cubrían un amplio espectro de diez dimensiones. Segundo, los investigadores elaboraron un cuestionario revisado por maestros tutores, especialistas y profesionales, que mejoraron la claridad y adecuación de los ítems. Al principio del CI había una declaración introductoria que indicaba su propósito y significado, así como una garantía de confidencialidad. La segunda sección contenía preguntas abiertas con respecto a la información demográfica de profesores y estudiantes, incluidas preguntas sobre género, edad, años de experiencia docente y de formación en educación especial. La tercera sección constaba de 53 declaraciones agrupadas en cinco dimensiones para evaluar las competencias de los docentes en EI. Los encuestados respondieron en una escala Likert de 4 puntos que iban desde "nunca" a "siempre". Finalmente, la cuarta sección, compuesta por 6 ítems, distinguía entre el uso y la valoración de los recursos tecnológicos para el estudiante, cada una con una escala de medida de las respuestas de 4 puntos que iban desde "nunca" a "siempre" para medir la frecuencia de uso y desde "muy adecuado" a "muy inadecuado" para medir la valoración de su uso. La validez de contenido del cuestionario se hizo a través del juicio de expertos (maestros tutores, especialistas y grupos profesionales) que estimaron la idoneidad de las declaraciones seleccionadas del $\mathrm{Cl}$.

Los 59 ítems de Cuestionario Incluye $(\mathrm{Cl})$ tienen concomitancias con los 40 ítems de Inclusive Teachers Competency Questionnaire (ITCQ), que mide las competencias de los maestros regulares de la competencia de El (Deng, Wang, Guan y Wang, 2017, p. 418); igualmente, con los 20 ítems de The Scale of Teachers' Attitudes Toward Inclusive Classrooms (STATIC), utilizado por Priyadarshini y Thangarajathi (2016-2017, p. 31), para medir las actitudes docentes hacia la El; con las 38 declaraciones de School Participant Empowerment Scale (Moran y Larwin, 2017, p. 10), que mide la percepción global de empoderamiento de profesores y directores; con el cuestionario de 15 destrezas Teachers' Preparation to Demonstrate Skills Associated With Inclusive Education and Collaboration de Zagona, Kurth, y MacFarland (2017, p. 166), usado para conocer el grado de 
preparación de los maestros para demostrar destrezas asociadas con la El; con la escala de 15 ítems The Sentiments, Attitudes, and Concerns about Inclusive Education Revised (SACIER-R) y de 18 ítems Teacher Efficacy for Inclusive Practice (TEIP) Scale para medir la adopción de estrategias para promover la inclusión de los estudiantes, la colaboración y la gestión de conductas, diseñada por Sharma, Loreman y Forlin (2012, pp. 18-19) y usadas por Özokcu (2018, p. 8) y Chao, Lai, Ji, Lo y Sin (2018, p. 166), entre otros; en parecidos términos se habían aplicado los 45 ítems del Cuestionario sobre Actitudes hacia la Inclusión e Interculturalidad (CAII) (Alegre y Villar, 2015, p. 17) para medir las actitudes de los alumnos de la titulación de maestro hacia la El, las traducciones al alemán de cuatro escalas: Attitudes towards Inclusion Scale (AIS), Intention to Teach in an Inclusive Classroom Scale (ITICS), Concerns about Inclusive Education Scale (CIES) y Teacher Efficacy for Inclusive Practice (TEIP) Scale, aplicadas por Miesera, DeVries, Jungjohann, y Gebhardt, 2018, pp. 2-3) o el Educational Leader Questionnaire to Determine SelfAuthoring and/or Self-Transforming Traits of Teachers, adaptado por Sprott (2019, p. 328).

\section{Análisis de datos}

Se codificaron los datos del $\mathrm{Cl}$ e introdujeron en el Statistical Package for Social Sciences (SPSS) para Mac. Como los datos de las actitudes, competencias y acciones profesionales de los docentes no se habían examinado previamente en estudios empíricos, se analizaron los datos a través de un análisis factorial exploratorio (AFE) contenido en el SPSS para determinar grupos de variables significativas latentes existentes entre ítems del $\mathrm{Cl}$ (por ejemplo, un grupo interrelacionado de ítems del $\mathrm{Cl}$ ). AFE es una técnica estadística que permite la reducción del tamaño de un banco de ítems con objeto de identificar las dimensiones profesionales básicas de los enseñantes de este estudio, y así ha sido utilizado por otros investigadores que han utilizado el método de investigación basado en el sondeo (Deng et al., 2017).

Desde el punto de vista técnico, los factores son construcciones o dimensiones latentes que hemos usado para declarar las relaciones de empoderamiento de una serie de variables, de forma que se representen las variables observadas $\mathrm{p}$ del $\mathrm{Cl}$ con una nueva serie $\mathrm{m}$ derivada de variables de empoderamiento, de tal manera que $\mathrm{m}$ sea $<$ que $\mathrm{p}$.

\section{Resultados}

Usamos dos métodos cuantitativos para evaluar la matriz de correlaciones. Uno consistió en el análisis de la adecuación de la muestra de Kaiser-Meyer-Olkin (KMO), cuyo valor obtenido (.766) sugirió que la matriz de intercorrelaciones se podía tratar con el análisis factorial. El test de esfericidad de Bartlett confirmó el resultado anterior, que fue significativo $\left[\mathrm{X}^{2}(2080)=22006,100, \mathrm{p}<.001\right]$ indicando que la matriz de correlaciones era factorizable, es decir, que la muestra se adecuaba globalmente e ítem a ítem a la realización del AFE. El alpha de Cronbach dio un coeficiente de .841, que maximizaba la generalizabilidad de los factores. Con el fin de determinar el número de factores necesarios para representar los datos, examinamos el porcentaje de la varianza total que es explicada por cada uno de ellos, obteniéndose en nuestro caso que siete factores explican el 58,063 de dicha varianza (Tabla 1). 
Tabla 1. Varianza total explicada

\begin{tabular}{|c|c|c|c|c|c|c|c|c|c|}
\hline \multirow[t]{2}{*}{ Factor } & \multicolumn{3}{|c|}{ Autovalores iniciales } & \multicolumn{3}{|c|}{$\begin{array}{l}\text { Sumas de las saturaciones al } \\
\text { cuadrado de la extracción }\end{array}$} & \multicolumn{3}{|c|}{$\begin{array}{c}\text { Suma de las saturaciones al cuadrado } \\
\text { de la rotación }\end{array}$} \\
\hline & Total & $\begin{array}{r}\% \text { de la } \\
\text { varianza }\end{array}$ & $\begin{array}{c}\% \\
\text { acumulado }\end{array}$ & Total & $\begin{array}{c}\% \text { de la } \\
\text { varianza }\end{array}$ & $\%$ acumulado & Total & $\begin{array}{l}\% \text { de la } \\
\text { varianza }\end{array}$ & $\%$ acumulado \\
\hline 1 & 15,263 & 23,481 & 23,481 & 13,550 & 20,846 & 20,846 & 9,758 & 15,012 & 15,012 \\
\hline 2 & 8,516 & 13,101 & 36,582 & 8,717 & 13,411 & 34,257 & 9,028 & 13,889 & 28,901 \\
\hline 3 & 6,016 & 9,255 & 45,837 & 5,661 & 8,709 & 42,966 & 5,351 & 8,232 & 37,133 \\
\hline 4 & 3,303 & 5,082 & 50,919 & 3,036 & 4,671 & 47,637 & 4,329 & 6,659 & 43,793 \\
\hline 5 & 3,181 & 4,894 & 55,812 & 2,735 & 4,207 & 51,845 & 3,319 & 5,107 & 48,899 \\
\hline 6 & 2,695 & 4,146 & 59,959 & 2,189 & 3,368 & 55,213 & 3,045 & 4,684 & 53,584 \\
\hline 7 & 2,121 & 3,263 & 63,222 & 1,853 & 2,850 & 58,063 & 2,912 & 4,479 & 58,063 \\
\hline 8 & 1,975 & 3,038 & 66,260 & & & & & & \\
\hline 9 & 1,762 & 2,711 & 68,971 & & & & & & \\
\hline 10 & 1,639 & 2,521 & 71,493 & & & & & & \\
\hline 11 & 1,363 & 2,097 & 73,590 & & & & & & \\
\hline 12 & 1,219 & 1,876 & 75,465 & & & & & & \\
\hline$\ldots$ & $\ldots$ & $\ldots$ & $\ldots$ & & & & & & \\
\hline 65 &, 014 &, 021 & 100,000 & & & & & & \\
\hline
\end{tabular}

Se eligió, por tanto, una estructura factorial de siete factores que explicarían el $58,063 \%$ de la varianza. Esta decisión se refleja también en el gráfico de sedimentación (Figura 1) donde los factores se indican en el eje de abscisas y los ítems en las ordenadas apreciándose visualmente los siete factores que poseen las varianzas más altas.

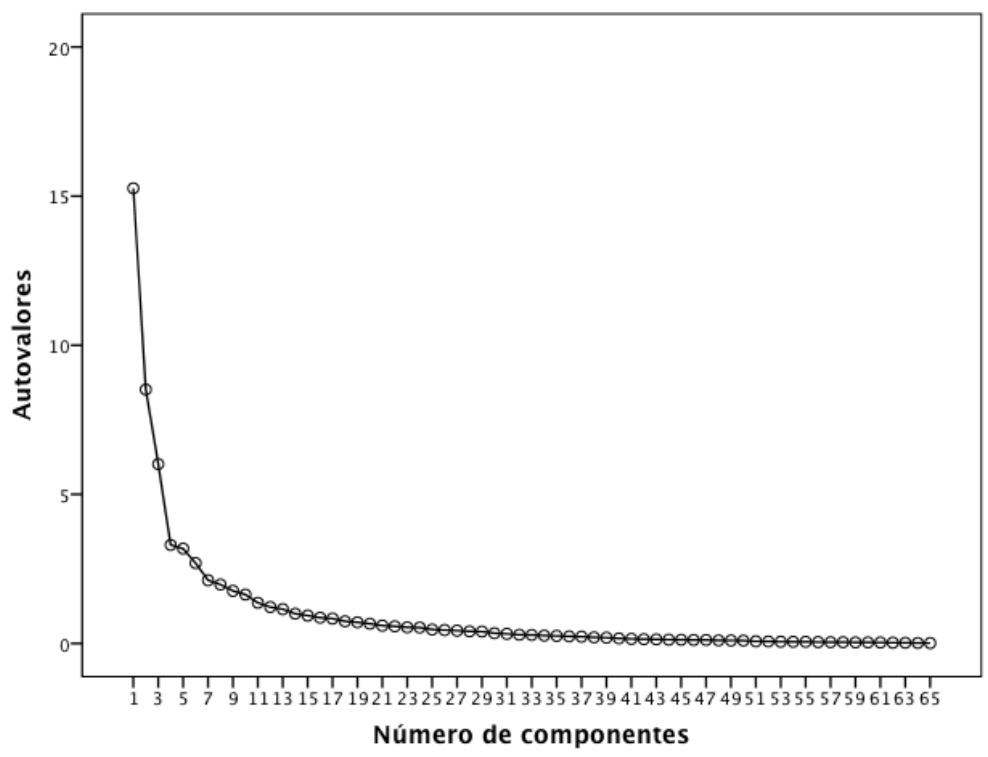

Figura 1. Gráfico de sedimentación 
Las comunalidades fueron muy altas (salvo algunos ítems excepcionales que fluctúan entre .700 y .998) lo que implica que todos los ítems estaban muy bien representados en el espacio de los factores. Realizado el análisis factorial de componentes principales, se pudo simplificar la información mediante la matriz de correlaciones que nos permitió responder a la pregunta de por qué unos ítems se relacionan más entre sí y menos con otros, lo que nos llevó a intentar conocer la estructura subyacente a los mismos. La matriz rotada siguiendo el método ortogonal varimax de máxima verosimilitud nos permitió interpretar e identificar los factores sustancialmente significativos al agrupar los ítems que tienen una carga grande respecto al mismo factor. Los pesos más próximos a cero se consideran bajos y los más cercanos a uno permitieron definir los factores.

Al interpretar los factores se necesita observar los pesos que determinan la fuerza de las relaciones. Considerando el tamaño de la muestra (297 profesores) y la facilidad de interpretación de las variables, decidimos elegir como corte una carga $<.40$ para los factores (Yon y Pierce, 2013, 84-85).

En la rotación de los factores se ha considerado la solución planteada por Thurstone (1928) del principio de estructura simple según el cual la matriz de cargas factoriales ha de reunir tres características: 1. Cada factor debe tener unos pocos pesos altos y los demás próximos a cero; 2 . Cada ítem no debe estar saturado más que en un factor; 3. No deben existir factores con la misma distribución (dos factores distintos deben presentar distribuciones diferentes de cargas altas y bajas).

Factor 1: Desarrollo Profesional (DP) sin rotación tiene un poder explicativo del $15.012 \%$ de la varianza total, obteniendo el ítem 30 el mayor peso ("Considero que debo recibir formación en conocimientos específicos del implante coclear"), representando el 73,788\% (.8592), seguido del ítem 31 ("Considero que debo recibir formación en las medidas organizativas a tomar en mi centro"), que significa el 70,089\% (.8422) o el ítem 9 ("Un proceso de atención a la diversidad de calidad requiere de experiencia previa con alumnos con necesidades educativas"), que simboliza el $66,748 \%$ (.8172). Le siguen los ítems 22, 32 y 21 que suponen respectivamente el $58,643 \%$, el $54,316 \%$ y el 52,417\%, como ítems más destacados.

Factor 2: Autoeficacia en la enseñanza de destrezas. Supone el 13,889\% de la varianza total. Destacamos el ítem 51 ("Ha mejorado la atención del alumnado con IC/AUD cuando escucha en actividades en pequeños grupos") que tiene el mayor peso con 80,046\% (.8972), y le sigue el ítem 48 ("Ha mejorado en la comprensión de los comentarios de los compañeros en clase de los niños con IC/AUD"), cuyo peso representa el 78,676\% (.8872). A continuación, ocho ítems con valores por encima de $65 \%$.

Factor 3: Liderazgo inclusivo. Supone el 8,232\% de la varianza total. Hay cuatro ítems (4, 3, 29 y 2) que tienen un peso por encima del valor .614. Seleccionamos, como ejemplo ilustrativo, el ítem 4: "La atención a la diversidad debe ocupar un papel relevante en mi práctica docente".

Factor 4: Gestión de la tecnología. Supone el 6,659\% de la varianza total. Con valores más bajos que en los factores anteriores, destacan aquí los ítems 57, 55 y 54 . 
Un caso ilustrativo es el ítem 57 ("Opinión sobre el uso de la tecnología en el aula: pizarra digital"), que representa el 35,760\% (-.5982).

Factor 5: Retos de implicación familiar. Supone el 5,107\% de la varianza total. Resaltamos el ítem 35 ("Sus padres acuden a las reuniones convocadas por los tutores"), que tiene el mayor peso representando el 36,844\% (.6072).

Factor 6: Marcos de pensamiento experto. Supone el 4,684\% de la varianza total. Destacan los ítems 18 ("Hago esquemas de seguimiento en la pizarra") y 19 ("Escribo en la pizarra las palabras clave o ideas principales") con mayor peso en el factor, que representan el 54,022\% (.7352) y el 51,552\% (.7182), respectivamente.

Factor 7: Usabilidad tecnológica y accesibilidad universal. Supone el 4,479\% de la varianza total. Los ítems 65 ("Valoración tecnología en el aula: apoyos visuales") y 64 ("Valoración tecnología en el aula: imágenes / presentaciones con imágenes") suponen el mayor peso en el factor representando el $66,992 \%(.8752)$ y el $75,342 \%$ (.8682) respectivamente.

\section{Discusıón}

Los hallazgos de este estudio indican que $\mathrm{Cl}$ es un cuestionario que mide el empoderamiento de maestros en centros de El. El primer factor lo etiquetamos Desarrollo Profesional (DP). Contiene cinco ítems sobre formación necesaria, cinco sobre formación recibida, cinco sobre la aplicación de la formación recibida, y uno sobre la inclusión y los recursos. Subrayamos en este factor el tópico del conocimiento (necesario y adquirido), las medidas organizativas necesarias a nivel de clase y de centro, y la aparente contradicción en las opiniones que tienen los maestros sobre el apoyo institucional para asistir a actividades formativas (Waitoller y Artiles, 2013).

Designamos Autoeficacia en la enseñanza de destrezas al segundo factor. Incluye 12 ítems, y mide la dificultad que tienen los estudiantes en destrezas de escucha/audición, subrayando los maestros la comprensión como mejor resultado de su enseñanza, así como otras habilidades percibidas en los niños y adolescentes con dificultades auditivas: mejora de la atención, comprensión de comentarios, implicación social en discusiones, respuestas apropiadas a preguntas, seguimiento rápido de instrucciones, discriminación, ritmo de aprendizaje y mantenimiento en la tarea durante más tiempo. La eficacia de esas competencias profesionales se asocia con actitudes positivas hacia la inclusión. Todo lo contrario ocurrió en el estudio de Chao et al. (2018), que consideraron la enseñanza inclusiva de los maestros de educación primaria y la gestión de la conducta de los profesores de educación secundaria como las acciones profesionales menos eficaces, medidas por la Teacher Efficacy for Inclusive Practice (TEIP) Scale (Sharma, Loreman y Forlin, 2012).

El tercer factor lo rotulamos Liderazgo inclusivo. Tiene ocho ítems que se resumen en las siguientes ideas clave: afrontar el reto de la diversidad, responsabilidad del profesorado competente, trabajar de manera colaborativa, y ofrecer servicios y recursos tecnológicos adecuados y suficientes (León, Crisol y Moreno, 2018). 
El cuarto factor, que denominamos Gestión de la tecnología, reduce a tres el número de ítems sobre inclusión y recursos: sistema roger, bucle magnético, pizarra digital. Esto significa que el profesorado visiona los recursos tecnológicos como factores para la comunicación en clase. Un ambiente de escucha es esencial para los estudiantes con dificultades auditivas y los recursos ponderados por los maestros mejoran las percepciones de los estudiantes. Indudablemente una clase ruidosa de altos decibelios perturba la percepción de los eventos sonoros de una clase, porque la calidad del sonido crea actitudes positivas en los estudiantes, principalmente varones, para usar tecnologías asistidas (Rekkedal, 2012).

El quinto factor, Retos de implicación familiar, abarca siete ítems sobre la complicidad de la institución familiar: los maestros tutores opinan que las familias solicitan y acuden a las reuniones, porque dan importancia al rendimiento académico de sus hijos, participan en las actividades del centro y supervisan las tareas escolares de sus hijos. Padres y madres por igual se ocupan indistintamente de los asuntos académicos de sus hijos. No consta que las familias hayan pasado por estados de estrés, como ocurrió en el estudio de Zaidman-Zait, Most, Tarrasch, Haddad-eid y Brand (2016), que reconocieron que las familias habían solventado desafíos como la aceptación social de los hijos o la autoeficacia de los padres.

El sexto factor, Marcos de pensamiento experto, está compuesto por 10 ítems sobre la aplicación de la formación recibida. Los maestros opinaron sobre lo que hicieron en las aulas para trasladar la teoría a la práctica. Los ítems se agrupan en representaciones que sintetizan una educación experta, según Zierer (2015). Los cuatro pilares didácticos son los siguientes: estrategias metodológicas (los maestros realizan esquemas de seguimiento, escriben en la pizarra las palabras clave, preparan actividades, verifican el entendimiento de los alumnos, y utilizan el cañón o proyector); comunicación (los maestros utilizan un vocabulario y lenguaje sencillo, utilizan gestos o lengua de signos); planificación de la enseñanza (los maestros eligen materiales con alto contenido visual, fijan los contenidos básicos y complementarios), y evaluación del alumnado (los maestros adaptan los exámenes o pruebas de evaluación).

Finalmente, el séptimo factor incluye la evaluación de la tecnología en el aula. Los seis factores insinúan Usabilidad tecnológica y accesibilidad universal. El uso y el acceso a apoyos visuales, imágenes/presentaciones, pizarra digital, bucle magnético, sistema roger y fm tienen como meta generar medios personalizables que faciliten y motiven el desarrollo físico, cognitivo y social de los estudiantes con dificultades auditivas. Las técnicas anteriormente mencionadas constituyen la base de un sistema de tecnología inteligente de eSalud y eCuidado, con las que se podría resolver los problemas en niños y adolescentes con dificultades auditivas derivados de la falta de atención en ambientes ruidosos, de las dificultades para mantener interacciones sociales, y de la costumbre decreciente de participar en actividades extracurriculares (King, Boutilier y Chorne, 2018). 


\section{Limitaciones}

Este estudio descriptivo ha soslayado observaciones sistemáticas y entrevistas a los maestros. Además, ha obviado el conocimiento de los factores resultantes por género. Consecuentemente se desconoce la existencia de igualdad o diferencia de elementos de empoderamiento en las mujeres, independientemente de que las maestras fueran mayoritarias en la muestra (82.2\%).

\section{Recomendaciones y desarrollo futuro}

Los hallazgos de este estudio tienen implicaciones teóricas para comprender las competencias de empoderamiento y emprendimiento que tienen los maestros de las escuelas de integración de niños y adolescentes con IC o AUD en el servicio público de Islas Canarias. Reflexionando sobre los factores hallados, se pueden planificar y desarrollar estrategias para la individualización de la instrucción y adaptar estándares de aprendizaje para estudiantes con dificultades auditivas, y paralelamente sugerir acciones formativas para el Prácticum de la titulación de Maestros. Los equipos directos que apoyen la colaboración con maestros tutores y especialistas en audición y lenguaje sentarían los fundamentos del empoderamiento en los centros educativos. En el futuro del área de empoderamiento de maestros de escuelas inclusivas se deberá relacionar los factores obtenidos con su grado de satisfacción profesional. Asimismo, comparar los factores de tutores del sector público obtenido con otros que se investigaran en maestros del sector privado (Moran y Larwin, 2017).

\section{Conclusiones}

Los factores Desarrollo profesional (DP), Autoeficacia en la enseñanza de destrezas, Liderazgo inclusivo, Gestión de la tecnología, Retos de implicación familiar, Marcos de pensamiento experto, y Usabilidad tecnológica y accesibilidad universal procedentes de $\mathrm{CI}$ han sido desarrollados y validados con fiabilidad y validez satisfactorias. Los factores integran un modelo conceptual coherente con un liderazgo inclusivo por su visión y misión de la escuela orientada a la resolución de problemas de la comunidad educativa (León, Crisol y Moreno, 2018). Bajo la perspectiva profesional docente, los siete factores de inclusividad son nucleares para la enseñanza inclusiva en otros contextos geográficos españoles y comunes a lo que se espera de los maestros regulares de otros ámbitos culturales (Liakopoulou, 2011; Sprott, 2019).

\section{REFERENCIAS BIBLIOGRÁFICAS}

Alegre, O. M. y Villar, L. M. (2015). Inclusión e interculturalidad. Un estudio en el marco de la enseñanza universitaria. Revista nacional e internacional de educación inclusive, 8(3), 10-12.

Amin, M., Till, A. y McKimm, J. (2018). Inclusive and person-centred leadership: creating a culture that involves everyone. British Journal of Hospital Medicine, 79(7), 402-407. https://doi.org/10.12968/hmed.2018.79.7.402. 
Archbold, S. y Mayer, C. (2012). Deaf Education: The Impact of Cochlear Implantation? Deafness and Education International, 14(1), 2-15. https://doi.org/10.1179/1 557069X12Y.0000000003.

Black, W. R. y Simon, M. D. (2014). Leadership for All Students: Planning for More Inclusive School Practices. International Journal of Educational Leadership Preparation, 9(2), 153-172.

Booth, T. y Ainscow, M. (2000). Index for inclusion. Bristol: Centre for Studies on Inclusive Education.

Bruce, S., Dinatale, P. y Ford, J. (2008). Meeting the needs of deaf and hard of hearing students with additional disabilities through professional teacher development. American Annals of the Deaf, 153(4), 368-375.

Caselli, M. C., Rinaldi, P., Varuzza, C., Giuliani, A. y Burdo, S. (2012). Cochlear implant in the second year of life: lexical and grammatical outcomes. Journal of Speech, Language, and Hearing Research, 55(2), 382-94. https://doi.org/10.1044/10924388(2011/10-0248.

Chao, C. N. G., Lai, F. T. T., Ji, M., Lo, S. K. y Sin, K. F. (2018). Which inclusive teaching tasks represent the highest level of teacher efficacy in primary and secondary schools? Teaching and Teacher Education, 75, 164-173. https://doi.org/10.1016/j. tate.2018.06.014.

Davenport, C.A. y Alber-Morgan, S.R. (2016). I Have a Child With a Cochlear Implant in My Preschool Classroom. Now What? Teaching Exceptional Children, September 2016. SAGE Publishing. https://doi.org/10.1177/0040059916651430.

De Boer, A., Pijl, S. J. y Minnaert, A. (2011). Regular primary schoolteachers' attitudes towards inclusive education: a review of the literatura. International Journal of Inclusive Education, 15(3), 331-353. https://doi.org/10.1080/13603110903030089.

Deng, M., Wang, S., Guan, W. y Wang, Y. (2017). The development and initial validation of a questionnaire of inclusive teachers' competency for meeting special educational needs in regular classrooms in China. International Journal of Inclusive Education, 21(4), 416-427. https://doi.org/10.1080/13603116.2016. 1197326.

Jachova, Z. y Karovska, A. (2008). Analyses of the Teacher-Pupil Interaction Within the Inclusive Classroom-Case Study of a Child With a Cochlear Implant. The Journal of Special Education and Rehabilitation, 9(3-4), 7-22.

Jachova, Z. y Kovacevic, J. (2010). Cochlear implants in the inclusive classroom: a case study. Support for Learning, 25, 33-37. https://doi.org/10.1111/j.14679604.2009.01436.x.

King, S., Boutilier, J. A. y Chorne, J. M. (2018). Managing Chronic Pain in the Classroom: Development and Usability Testing of an eHealth Educational Intervention for Educators. Canadian Journal of School Psychology, 33(2), 95-109. https://doi. org/10.1177/0829573516674308. 
León, M. J., Crisol, E. y Moreno, R. (2018). Las Tareas del Líder Inclusivo en Centros Educativos de Zonas Desfavorecidas y Favorecidas. REICE. Revista Iberoamericana sobre Calidad, Eficacia y Cambio en Educación, 16(2), 21-40. https://doi. org/10.15366/reice2018.16.2.002.

Liakopoulou, M. (2011). The Professional Competence of Teachers: Which Qualities, Attitudes, Skills and Knowledge Contribute to a Teacher's Effectiveness. International Journal of Humanities and Social Science, 1, 66-78.

Maskit, D. (2011). Teachers' attitudes toward pedagogical changes during various stages of professional development. Teaching and Teacher Education, 27(5), 851860. https://doi.org/10.1016/j.tate.2011.01.009.

Mather, J., Archbold, S. y Gregory, S. (2011). Deaf Young People with Sequential Bilateral Cochlear Implants: The Experience of Parents and Teachers. Deafness and Education International, 13(4), 173-198. https://doi.org/10.1179/1557069X1 1Y.0000000009.

Mayer, C. y Trezek, B. J. (2018). Literacy Outcomes in Deaf Students with Cochlear Implants: Current State of the Knowledge. The Journal of Deaf Studies and Deaf Education, 23(1), 1-16. https://doi.org/10.1093/deafed/enx043.

Miesera, S., DeVries, J. M., Jungjohann, J. y Gebhardt, M. (2018). Correlation between attitudes, concerns, self-efficacy and teaching intentions in inclusive education evidence from German pre-service teachers using international scales. Journal of Research in Special Educational Needs, 19(2), 103-114. https://doi. org/10.1111/1471-3802.12432.

Moeller, M. P., Carr, G., Seaver, L., Stredler-Brown, A. y Holzinger, D. (2013). Best Practices in Family-Centered Early Intervention for Children Who Are Deaf or Hard of Hearing: An International Consensus Statement. Journal of Deaf Studies and Deaf Education, 18(4), 429-445.

Montgomery, A. y Mirenda, P. (2014). Teachers' self-efficacy, sentiments, attitudes and concerns about the inclusion of students with developmental disabilities. Exceptionality Education International, 24, 18-32.

Moran, K. y Larwin, K. H. (2017). Building Administrator's Facilitation of Teacher Leadership: Moderators Associated with Teachers' Reported Levels of Empowerment. Journal of Organizational and Educational Leadership, 3(1), Article 2.

Özokcu, O. (2018). The Relationship Between Teacher Attitude and Self-Efficacy for Inclusive Practices in Turkey. Journal of Education and Training Studies, 6(3), 6-12. https://doi.org/10.11114/jets.v6i3.3034.

Priyadarshini, S. S. y Thangarajathi, S. (2016-2017). Effect of Selected Variables on Regular School Teachers Attitude towards Inclusive Education. Journal on Educational Psychology, 10(3), 28-38.

Punch, R. y Hyde, M. (2011). Social Participation of Children and Adolescents With Cochlear Implants: A Qualitative Analysis of Parent, Teacher, and Child Interviews. 
Journal of Deaf Studies and Deaf Education, 16(4), 474-93. https://doi.org/10.1093/ deafed/enr001.

Rae, D. y Carswell, M. (2001). Towards a conceptual understanding of entrepreneurial learning. Journal of Small Business and Enterprise Development, 8(2), 150-158. https://doi.org/10.1108/EUM0000000006816.

Rekkedal, A. M. (2012). Assistive Hearing Technologies Among Students With Hearing Impairment: Factors That Promote Satisfaction. The Journal of Deaf Studies and Deaf Education, 17(4), 499-517. https://doi.org/10.1093/deafed/ens023.

Schraer-Joiner, L. y Prause-Weber, M. (2009). Strategies for Working with Children with Cochlear Implants. Music Educators Journal, 96(1), 48-55.

Sharma, U., Loreman, T. y Forlin, C. (2012). Measuring teacher efficacy to implement inclusive practices. Journal of Research in Special Educational Needs, 12(1), 12 21. https://doi.org/10.1111/j.1471-3802.2011.01200.x.

Sprott, R. A. (2019). Factors that foster and deter advanced teachers' professional development. Teaching and Teacher Education, 77, 321-331. https://doi. org/10.1016/j.tate.2018.11.001.

Thurstone, L. L. (1928). Attitudes can be measured. American Journal of Sociology, 33, 529-554. https://doi.org/10.1086/214483.

UNESCO (1994). The Salamanca Statement and Framework for Action on Special Needs Education. Paris: UNESCO/Ministry of Education, Spain. (ED-34/WS/18).

Van Mieghem, A., Verschueren, K., Petry, K. y Struyf, E. (2018). An analysis of research on inclusive education: a systematic search and meta review. International Journal of Inclusive Education, 1-15. https://doi.org/10.1080/13603116.201 8.1482012 .

Vermeulen, A., De Raeve, L., Langereis, M. y Snik, A. (2012). Changing Realities in the Classroom for Hearing-Impaired Children with Cochlear Implant. Deafness and Education International, 14(1), 36-47.

Waitoller, F. R. y Artiles, A. J. (2013). A Decade of Professional Development Research for Inclusive Education. A Critical Review and Notes for a Research Program. Review of Educational Research, 83(3), 319-356. https://doi. org/10.3102/0034654313483905.

Walker, E. A. y McGregor, K. K. (2013).Word learning processes in children with cochlear implants. Journal of Speech, Language, and Hearing Research, 56(2), 375-87.

Wilson, K., Ellen, M. y Houston, K. T. (2010). Professional Development for In-Service Practitioners Serving Children who are Deaf and Hard of Hearing. The Volta Review, 110(2), 231-247.

Yong, A. G. y Pearce, S. (2013). A Beginner's Guide to Factor Analysis: Focusing on Exploratory Factor Analysis. Tutorials in Quantitative Methods for Psychology, 9(2), 79-94. https://doi.org/10.20982/tqmp.09.2.p079. 
Zagona, A. L., Kurth, J. A. y MacFarland, S. Z. C. (2017). Teachers' Views of Their Preparation for Inclusive Education and Collaboration. Teacher Education and Special Education, 40(3), 163-178. https://doi.org/10.1177/0888406417692969.

Zaidman-Zait, A., Most, T., Tarrasch, R., Haddad-eid, E. y Brand, D. (2016). The Impact of Childhood Hearing Loss on the Family: Mothers' and Fathers' Stress and Coping Resources. The Journal of Deaf Studies and Deaf Education, 21(1), 23-33. https:// doi.org/10.1093/deafed/env038.

Zierer, K. (2015). Educational expertise: the concept of 'mind frames' as an integrative model for professionalisation in teaching. Oxford Review of Education, 41(6), 782798. https://doi.org/10.1080/03054985.2015.1121140. 